\title{
How important is roaming in the photodegradation of nitrobenzene?
}

\author{
Angelo Giussani, ${ }^{1, \dagger}, *$ Graham A. Worth ${ }^{1}$ \\ ${ }^{1}$ Department of Chemistry, University College London, 20 Gordon Street, \\ London WC1H 0AJ, UK.
}

${ }^{\dagger}$ Current address: Instituto de Ciencia Molecular, Universidad de Valencia, Catedrático José Beltrán 2, 46980 Paterna, Spain.

* E-mail: angelo.giussani@uv.es

At low excitation energies nitrobenzene photoreleases NO with low translational and rotational energy, while at higher excitation energies NO is photoreleased with both low and high translational and rotational energy. The fast products are formed through a singlet-triplet crossing (STC) region featuring an oxaziridine ring, while a ground state roaming mechanism was suggested to produce the slow molecules. Computing translational and rotational energies performing CASSCF classical dynamics, we here prove how the same oxaziridine STC can account for both fast and slow photoproducts, depending on the region of the seam through which the ground state is populated. A roaming-type STC/CI has also been characterized, from which slow NO molecules can also be formed through a roaming photodegradation mechanism, here in the excited state. The higher accessibility of the oxaziridine STC mechanism, $1.53 \mathrm{eV}$ lower in energy than the roaming path, questions the contribution of roaming in nitrobenzene NO photoproduction.

\section{Introduction}

Nitrobenzene is the smallest nitroaromatic compound, and is consequently the starting point in the characterization of this class of molecules, whose importance spans the field of energetic materials, ${ }^{1}$ the drug delivery sector, ${ }^{2}$ and the study of common urban air pollutants. ${ }^{3}$ Nitroaromatic compounds are also linked to basic research since they display properties that challenge fundamental photophysical/photochemical concepts, as the possibility of ultrafast intersystem crossings in organic molecules (1 nitronaphthalene is the organic compound with the fastest multiplicity change, around $100 \mathrm{fs}) .^{4-7}$

Nitrobenzene is characterized by a rich photochemistry. While most nitroaromatic compounds, including nitrobenzene itself, are know to photodegradate into nitric oxide (NO) and the corresponding aryloxy radical, ${ }^{8-10}$ nitrobenzene also photodegrades along two additional paths: one leading to nitrogen dioxide, the other resulting in atomic oxygen. ${ }^{11,12}$ The photodegradation of nitrobenzene to form NO has been proven to occur via two mechanisms. ${ }^{13,14}$ Employing multimass ion imaging techniques on nitrobenzene vapor, Lin et al. have determined a bimodal distribution of the translational energy of the NO molecules, which points to the presence of two different paths leading to NO photorelease. ${ }^{13}$ The bimodal distribution was observed exciting the system at 248 and $193 \mathrm{~nm}$ (5.00 and $6.42 \mathrm{eV}$ ), while employing a $266 \mathrm{~nm}(4.66 \mathrm{eV})$ wavelength only the slow component was observed. The fast component was more pronounced at the highest excitation wavelength employed.

This bimodal distribution was later confirmed by Suits and co-workers, who performed a stateselected direct current slice imaging study after excitation at $226 \mathrm{~nm}(5.49 \mathrm{eV}) .{ }^{14}$ In their work it was determined that the low translational energy products are characterized by low rotational energy, while the high translation energy molecules display high rotational energy. The two paths were theoretically investigated, arriving at the hypothesis that both processes pass through the photoisomerization of the nitro into a nitrite group followed by a thermally activated release of NO. 
For the fast molecule, however, the photoisomerization is mediated by the formation of an oxaziridine ring on the triplet states, as previously suggested by Lin et al. and by Zewail and coworkers and documented for other nitroaromatic systems, ${ }^{13,15-17}$ while for the slow product the photoisomerization is suggested to occur on the ground state according to a roaming mechanism.

Roaming reactions are a class of mechanisms not described in transition state theory, in which the molecule undergoes a frustrated dissociation to radicals. The formed fragments do not separate completely, but instead one of the two starts to "roam" in the field of the other, eventually reorientating and re-combining with the other into a different chemical entity. ${ }^{18}$ This type of mechanism has been proved to occur on the ground electronic state in molecules as $\mathrm{H}_{2} \mathrm{CO}$ and $\mathrm{CH}_{3} \mathrm{CHO},{ }^{19,20}$ while the possibility of roaming in the excited states has been shown for $\mathrm{NO}_{3}{ }^{21,22}$

The two mechanisms suggested by Suits and co-workers nicely match the original proposal of Chapman et al. on the nitro-to-nitrite photoisomerization process preceding NO photorelease in nitrated polycyclic aromatic hydrocarbons, in which the photoisomerization occurs according to two mechanisms: an intramolecular rearrangement mechanism characterized by the formation of an oxaziridine ring; and a dissociation-recombination mechanism characterized by a frustrated dissociation of the $\mathrm{CN}$ bond, closely resembling a roaming process. ${ }^{8}$

For nitrobenzene, Suits and co-worker presented what they defined as a roaming transition state, from which the corresponding photodegradation rates agree much better with the experimental data than the ones calculated from a tight transition state. Little information is however provided on the roaming transition state, and on how the molecule would be able to reach it, placed at more than 3 $\mathrm{eV}$ above the S0 minima.

Regarding its photophysics, ${ }^{17,23,24}$ different absorption bands have been reported for nitrobenzene in the gas-phase, ranging from 3.6 up to $7.6 \mathrm{eV}$. After UV absorption, nitrobenzene can rapidly decay into the ${ }^{1}\left(\mathrm{n}_{\mathrm{A}} \pi^{*}\right)$-S1 state. The ${ }^{1}\left(\mathrm{n}_{\mathrm{A}} \pi^{*}\right)$-S1 then mainly decays into the triplet manifold, reflected in the high triplet quantum yield $(>0.8),{ }^{25}$ populating the ${ }^{3}\left(\pi_{0} \pi^{*}\right)$ triplet state, which is coupled with ${ }^{1}\left(\mathrm{n}_{\mathrm{A}} \pi^{*}\right)$ by a particularly high spin-orbit coupling $\left(>60 \mathrm{~cm}^{-1}\right)$. The ${ }^{3}\left(\pi_{\mathrm{O}} \pi^{*}\right)$ can further relax into its minimum, ${ }^{3}\left(\pi_{\mathrm{O}} \pi^{*}\right)_{\min }$, or the ${ }^{3}\left(\mathrm{n}_{\mathrm{A}} \pi^{*}\right)$ state minimum, ${ }^{3}\left(\mathrm{n}_{\mathrm{A}} \pi^{*}\right)_{\min }$, from which a non-radiative decay through either a non-reactive (the $\left({ }^{3} n_{\mathrm{A}} \pi^{*} / \mathrm{gs}\right)_{\mathrm{STC2}}$ geometry of ref 17$)$ or a reactive (the (T1/S0) $)_{\text {stc-No }}$ geometry of ref 17) STC region constitutes the most probable final relaxation step, as reflected by the very low phosphorescence quantum yield $\left(<10^{-3}\right){ }^{26}$

In this contribution the mechanisms leading nitrobenzene to photorelease NO have been studied both performing static ab-initio CASSCF and CASPT2 computations and running on-the-fly classical dynamics on CASSCF potential energy surfaces. Three different paths have emerged, two related to the same STC region characterized by an oxaziridine structure and one ascribable to an excited state roaming mechanism. Using a simple classical model, the translational, rotational and vibrational energy of the formed NO have been derived, which in turn provides a tool to associate the characterized paths with either the formation of the slow or the fast NO molecules. On the basis of such a comparison and evaluating the probability of each path according to the energetic position of the corresponding key geometries, we conclude that both slow and fast products can actually originate from a non-roaming mechanism, consequently raising some doubt on the involvement of roaming in nitrobenzene NO photoformation.

\section{Methods}

\section{Computational details}

The static part of the study has been performed employing the well-tested CASPT2 and CASSCF methods $^{27-29}$ as implemented in the OpenMolcas software. ${ }^{30}$ Geometry optimizations of minima, 
minimum energy path (MEP), and minimum energy crossing points (MECP), have been performed at the CASSCF level. In all cases, the final energies have been computed at the CASPT2 level to take into account the dynamic correlation effects. No restrictions to the symmetry of the molecule have been imposed ( $\mathrm{C}_{1}$ symmetry). The basis set of atomic natural orbital (ANO) of L-type contracted to $\mathrm{C}, \mathrm{N}$ [4s,3p,1d]/H[2s1p] has been employed. ${ }^{31,32}$ An active space composed of 16 active electrons distributed in 13 active orbitals has been used in all final CASPT2 vertical calculations (see Figure S1-S6 and Figure S9 of reference 17), while a slightly reduced active space $(14,11)$ has been employed for all CASSCF geometry optimizations. The selected active spaces take into account both the $\pi$ nature of the system and the lone pairs of the oxygen atoms. The electronic structure of nitrobenzene is known to be challenging, ${ }^{33}$ but use of these active spaces appears adequate for the problem at hand, as indicated by our previous study on the system. ${ }^{17}$ All vertical calculations have been performed using state-averaging over the two roots of the same multiplicity, while S0 CASSCF dynamics have been performed computing only one root.

Within the CASPT2 calculations, an imaginary level-shift correction of 0.2 au has been used to minimize the effects of possible intruder states. The CASPT2 standard zeroth-order Hamiltonian has been used as originally implemented. ${ }^{28}$ The core orbitals have been frozen in the CASPT2 calculations. Such a CASPT2 approach has been validated in many different studies on organic molecules, providing a correct prediction, description, and interpretation of photophysical experimental data. ${ }^{29,34,35}$ In order to connect some important regions of the potential energy hypersurface $(\mathrm{PEH})$, linear interpolation of internal coordinate (LIIC) calculations have been performed. ${ }^{36}$ CASSCF MECPs have been optimized using the projected constrained optimization method as recently implemented in OpenMolcas. ${ }^{37}$ Transition state (TS) structures have been optimized using the saddle method as implemented in the OpenMolcas code. ${ }^{38}$ IRCs were run in order to verify the connection of the obtained TSs. The character of the obtained minima and TS structures has been confirmed by computing the corresponding CASSCF $(14,11)$ frequencies. In the regions of the potential energy hypersurfaces where two or more states of different spin multiplicity are degenerate, the spin orbit coupling (SOC) has been calculated as described elsewhere. ${ }^{39}$ The Cholesky decomposition has been used to speed up the calculation of two-electron integrals. ${ }^{40}$

In order to evaluate the ground state dynamics of the characterized static paths, CASSCF $(14,11)$ classical dynamics on the ground state PEH have been performed with initial zero velocity. The nuclei are moved according to the classical Newton's equations which are solved numerically using the velocity Verlet algorithm. A time step of either 1 or 10 a.u. (0.024 or $0.242 \mathrm{fs}$, respectively) has been used. In each case only a single trajectory has been performed. This approach is of incapable of providing averaged energies derived from a distribution of geometries describing a particular path and can consequently be better framed as an "improved minimum energy path" able to provide also velocities. All dynamics were performed using the OpenMolcas code. ${ }^{30}$

\section{Model for computing the NO translational, vibrational and rotational energy}

Using the data provided by the performed CASSCF $(14,11)$ classical dynamics, the following model was adopted in order to evaluate the kinetic, translational, vibrational and rotational energy of the NO molecule. Employing the computed velocities provided in the dynamics for each atom, the NO kinetic energy, $E_{k i n}$, has been computed as follow:

$$
E_{\text {kin }}=\frac{1}{2} * m_{O} * v_{O}^{2}+\frac{1}{2} * m_{N} * v_{N}^{2}
$$

The NO translational energy, $E_{t r}$, has been calculated as the kinetic energy of the center of mass of the NO fragment: 


$$
E_{t r}=\frac{1}{2} *\left(m_{O}+m_{N}\right) * v_{C M}^{2} ; \quad v_{C M}=\frac{m_{O} * v_{O}+m_{N} * v_{N}}{m_{O}+m_{N}}
$$

The NO vibrational energy, $E_{v i b}$, has been computed assuming that the NO bond is oscillating according to a harmonic oscillator, for which its energy results equal to

$$
E_{v i b}=\frac{1}{2} * \mu * \omega^{2} * A^{2} \quad ; \quad \omega=\frac{2 \pi}{T} ; \mu=\frac{m_{O} * m_{N}}{m_{O}+m_{N}}
$$

where $\mu$ is the reduced mass, $A$ is the maximum of the oscillation, and $\omega$ is the period. For each dynamics leading to NO formation, both $A$ and $T$ were derived from the graph obtained by plotting the NO bond distance as a function of time (Figure S15, S16, S17).

The NO rotational energy, $E_{\text {rot }}$, has finally been obtained after subtracting all other energy contributions to the NO kinetic energy:

$$
E_{r o t}=E_{k i n}-E_{t r}-E_{v i b}
$$

Such an analysis has been performed for all structures obtained along the first 145 fs after NO formation, which in turn is here considered starting from the structure in which the NO fragment is separated from the rest of the system by a distance equal or greater than $4 \AA$. The final energies reported in Table 2 have been computed averaging over all energies obtained at all the computed structures.

\section{Results and discussion}

\section{Paths leading to NO formation}

Starting from our previously obtained CASPT2 $(16,13)$ T1/S0 STC, ${ }^{17}{ }^{41}$ hereafter (T1/S0) $)_{\text {stc-No, from }}$ which the computation of the corresponding SO minimum energy path (MEP) evolves toward NO formation, classical dynamics on the S0 potential surface at the CASSCF $(14,11)$ level has been performed. In agreement with the static MEP result, the dynamics simulation leads to NO formation. As shown in Figure 1 the (T1/S0) stc-No geometry is characterized by an oxaziridine structure. As previously documented, ${ }^{17}$ (T1/S0) stc-No can be reached on the triplet manifold by surmounting a significant energy barrier of $1.32 \mathrm{eV}$, although the presence of more favorable decay paths yet to be determined are not excluded. It should be noted that the geometry is placed at 3.83

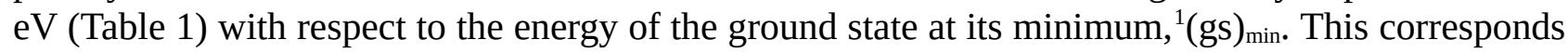
to a lower energy than any of the excitation frequencies employed in experimental works (which have been greater than $3.87 \mathrm{eV}$ ). ${ }^{11}$ The $\mathrm{T} 1$ and S0 states are coupled by a low value of the spin-orbit coupling (SOC), of around $5 \mathrm{~cm}^{-1}$, ascribable to the similar nature of the states (Figure S7). Despite the unfavorable energy barrier and the relatively low SOC, we consider the decay through the (T1/S0) stc-No structure as a possible path leading to NO photorelease, hereafter path1 (Figure 2), expected to be accessible at higher excitation energies.

To characterize the seam of intersection of the (T1/S0) $)_{\text {stc-No }}$ point, a T1/S0 minimum energy crossing point (MECP) computation has been performed starting from this structure at the CASSCF $(14,11)$ level. The CASSCF $(14,11)$ MECP structure reported in Figure 1, hereafter called (T1/S0) mecp, has been obtained, for which the degeneracy between $\mathrm{T} 1$ and $\mathrm{S} 0$ has been confirmed at the CASPT2 $(16,13)$ level (Table 1$)$. Similar to the $(\mathrm{T} 1 / \mathrm{S} 0)_{\text {stc-No }}$ geometry, a oxaziridine-like structure is recognizable in the (T1/S0) mecp structure, although the N7O9 distance has increased from the value of $1.662 \AA$ to $2.320 \AA$. A significant SOC value of $53 \mathrm{~cm}^{-1}$ now couples the states. This increased 
SOC value is probably associated with the increased closed shell character acquired here by the S0 but not the T1 state. From this new point, both a CASSCF $(14,11)$ ground state MEP and a CASSCF $(14,11)$ ground state classical dynamics simulation have been performed, both resulting in the formation of the same photoproduct, hereafter ${ }^{1}(\mathrm{~S} 0)_{\mathrm{ep}}$, characterized by the presence of an epoxide ring (Figure 1). The same path was also described in our previous work from a similar structure, $(\mathrm{T} 1 / \mathrm{S} 0)_{\text {stc-ep}}$, whose CASSCF MECP nature was not confirmed. ${ }^{17}$ At the CASPT2 $(16,13)$ level, the (T1/S0) stc-ep is actually $0.21 \mathrm{eV}$ lower in energy than the (T1/S0) mecp, reflecting the importance of dynamic correlation in geometry optimizations. For the sake of completeness, dynamics from the (T1/S0) stc-ep geometry has been performed, again resulting in the ${ }^{1}(\mathrm{~S} 0)_{\mathrm{ep}}$ structure. A $1.12 \mathrm{eV}$ energy barrier was estimated in order to reach (T1/S0) $)_{\text {stc-ep. }}$

As reported in the introduction, the final step in nitrobenzene NO photorelease is assumed to be a thermally activated ON bond breaking from the nitrite isomer. Another possibility, evaluated here, would be a thermally activated NO release from the ${ }^{1}(\mathrm{~S} 0)_{\mathrm{ep}}$ minimum. A transition state structure, hereafter ${ }^{1}(\mathrm{TS})_{\mathrm{ep}}$, connecting the ${ }^{1}(\mathrm{~S} 0)_{\mathrm{ep}}$ geometry with the photorelease of NO has been characterized. The nature of the ${ }^{1}(\mathrm{TS})_{\mathrm{ep}}$ point has been confirmed computing the corresponding CASSCF $(14,11)$ ground state frequencies (displaying one imaginary frequency related to the NO release) and the corresponding IRC path (Figure S8 and S9). The ${ }^{1}$ (TS) $)_{\text {ep }}$ is placed energetically 1.56 $\mathrm{eV}$ above the ${ }^{1}(\mathrm{~S} 0)_{\mathrm{ep}}$ minimum, corresponding to the energy barrier needed to be surmounted. Despite the significant barrier, the ${ }^{1}(\mathrm{TS})_{\mathrm{ep}}$ structure is placed $0.31 \mathrm{eV}$ below the (T1/S0) mecp geometry, for which a decay from the latter structure would provide enough energy to reach the ${ }^{1}(\mathrm{TS})_{\mathrm{ep}}$ transition state. From the ${ }^{1}(\mathrm{TS})_{\mathrm{ep}}$ geometry the corresponding ground state CASSCF $(14,11)$ classical dynamics evolves toward the ${ }^{1}(\mathrm{~S} 0)_{\mathrm{ep}}$ minimum, consequently highlighting that at ${ }^{1}(\mathrm{TS})_{\mathrm{ep}}$ the gradient points toward the epoxide product. Only from the second IRC converged point toward the formation of NO, the dynamics simulation proceeds toward NO formation. Globally, we consider the decay from the (T1/S0) mecp STC toward the ${ }^{1}(\mathrm{~S} 0)_{\text {ep }}$ minimum and the subsequent reaction on the ground state $\mathrm{PEH}$ passing through the ${ }^{1}(\mathrm{TS})_{\mathrm{ep}}$ transition state as a possible path towards NO photorelease, hereafter referred to as path2 (Figure 2).

Through a thorough exploration of the system PEHs, performing a series of CASSCF MECP optimizations imposing restrictions in the C1N7 bond distance and re-evaluating the energies at the CASPT2 level, a new T1/S0 STC region featuring a detachment of the nitro group, hereafter (T1/S0) roam, has been characterized. The structure is also a S0/S1 conical intersection (CI) and T1/S1 STC, since the three states (S0, S1, and T1) are degenerate (Table 1). At the (T1/S0) roam region the CASSCF $(16,13)$ S0 wave-function is mainly described by a single closed-shell configuration state function, while both $\mathrm{S} 1$ and $\mathrm{T} 1$ are mostly described by a single one-electron promotion from what can be recognized as the b2 orbital of the $\mathrm{NO}_{2}$ molecule to the $\sigma^{*}$ orbital associated with the original CN bond in nitrobenzene (Figure S10). ${ }^{42}$ The SOC between S0 and T1 at the (T1/S0) roam geometry is $48 \mathrm{~cm}^{-1}$. To evaluate the evolution on the S0 PEH, a CASSCF $(14,11)$ classical dynamics simulation on S0 starting from the (T1/S0) roam region has been performed, resulting in the formation of benzonitrite, which subsequently dissociates into NO and the corresponding aryloxy radical. This however cannot be taken as a reliable result, since along the dynamics a loss of the total energy greater than $0.1 \mathrm{eV}$ was observed. Additional simulations performed decreasing the integration time step still present the same problem, probably ascribable to the significant chemical change undertaken by the molecule.

From the (T1/S0) roam structure, a CASSCF $(14,11)$ S0 MEP has been performed, which terminates in the minima of the nitrite isomer, hereafter ${ }^{1}(\mathrm{~S} 0)_{\text {nitrite }}$ (Figure 1$)$, which can then been considered as

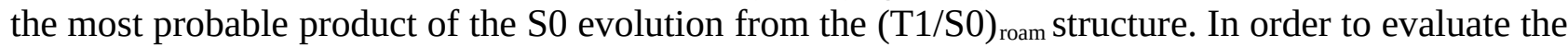
ability of the system to reach the (T1/S0) roam geometry, CASPT2 $(16,13)$ LIIC computations have been performed between the (T1/S0) roam point and the previously published ${ }^{1}\left(\mathrm{n}_{\mathrm{A}} \pi^{*}\right)_{\min }$ and ${ }^{3}\left(\mathrm{n}_{\mathrm{A}} \pi^{*}\right)_{\min }$ minima. ${ }^{17}$ Apart from the energy barrier derived from the energy difference between ${ }^{1}\left(\mathrm{n}_{\mathrm{A}} \pi^{*}\right)_{\min }$ and 


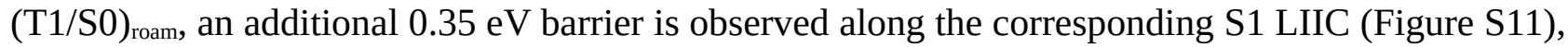
determining a global barrier separating the regions of $2.39 \mathrm{eV}$. No additional barrier is present between ${ }^{3}\left(\mathrm{n}_{\mathrm{A}} \pi^{*}\right)_{\min }$ and (T1/S0) roam (Figure S12), and the structures are separated by $2.44 \mathrm{eV}$. Despite such significant barriers, the computed LIICs show that the energetic requirement to reach the (T1/S0) roam region is mainly determined by its energetic position.

As explained in the introduction, the generally accepted hypothesis regarding nitrobenzene NO formation is that the final step involves a ground state release of NO from the nitrite isomer. Although this reaction has been the subject of different theoretical works, as far as the authors know a proper transition state describing the process has been elusive up to now. Such a transition state, hereafter ${ }^{1}$ (TS) nitrite, has been here identified at the $\operatorname{CASSCF}(14,11)$ level, proving its connection with the reaction through the computation of the corresponding frequencies and IRC path (Figure S13 and S14). It is significant to note that re-computing the energies of the structures along the forward CASSCF $(14,11)$ IRC path at the CASPT2 $(16,13)$ level, a slight increase in the energy of around $0.2 \mathrm{eV}$ instead of a descending path is obtained. The source of such a discrepancy could be due to the different description that the two methods provide of intermolecular interactions, ${ }^{43}$ as the ${ }^{1}(\mathrm{TS})_{\text {nitrite }}$ structure is characterized by an already significant separation of the NO fragment. Such a different description, although suggesting a possible source of errors in the present protocol, has not been observed for the preceding paths (see Figure S9, and Figure S11 of reference 17) leading toward NO formation. A full CASPT2 description has not here been obtained. The transition state can be considered energetically accessible, being placed $0.46 \mathrm{eV}$ above the ${ }^{1}(\mathrm{~S} 0)_{\text {nitrite }}$ minimum (Table 1). Both CASSCF $(14,11)$ MEP and classical dynamics from ${ }^{1}$ (TS) $)_{\text {nitrite }}$ have evolved toward

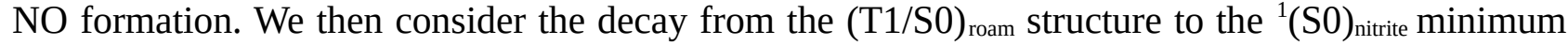
and the subsequent evolution through the ${ }^{1}$ (TS) nitrite transition state, as an additional path leading to NO photorelease, hereafter referred to as path3 (Figure 2), characterized by an initial roaming-like mechanism.

Despite not having elucidated the complete mechanism starting from light absorption, we have thus presented three different possible mechanisms leading to NO formation in nitrobenzene. These paths are summarized in Figure 2, and the energies of the relative critical points are reported in Table 1.

\section{Evaluation of the NO translational, vibrational and rotational energy}

For each of the three described paths, a CASSCF $(14,11)$ ground state classical trajectories from the critical points leading to NO formation have been performed. Such computations have proven the ability of the starting geometry to mediate NO production, and in addition have provided valuable data though which, using the model described in the Methods section, it was possible to evaluate both the total kinetic energy of the formed NO molecules, and the corresponding translational, vibrational and rotational contributions. The computed values are reported in Table 2, together with the available experimental data.

From the work of Lin et al. ${ }^{13}$ at the reported NO translational energy distribution after excitation at 266, 248 and $193 \mathrm{~nm}$ (4.66, 5.00, and $6.42 \mathrm{eV}$ ), see Figure 2, 4 and 6 in reference 13, it is possible to estimate that the NO translational energy peak is around $10 \mathrm{kcal} / \mathrm{mol}(0.43 \mathrm{eV})$ for the low translational energy products and at around $30 \mathrm{kcal} / \mathrm{mol}(1.30 \mathrm{eV})$ for the high translational energy molecules, although a continuum is actually visible between $5-40 \mathrm{kcal} / \mathrm{mol}(0.22-1.73 \mathrm{eV})$. These two peaks for the low and high NO translational energy nicely match the computed averaged translational energy of $\mathrm{NO}$ formed through path 2 and 1, equal to 0.53 and $1.24 \mathrm{eV}$, respectively (Table 2). Moreover the difference in their energy, $0.71 \mathrm{eV}$, is in reasonable agreement with the difference reported by $\mathrm{Lin}$ et al. in the peaks of slow and fast components from the photodissociation at $193 \mathrm{~nm}$, equal to $22 \mathrm{kcal} / \mathrm{mol}(0.95 \mathrm{eV})$. It is then plausible to associate the 
high and low translational energy NO detected by Lin et al. to the mechanism described by path 1 and 2, respectively, without a substantial involvement of a roaming process.

Table 1. CASPT2 $(16,13)$ energies $(\mathrm{eV})$ for the low-lying singlet and triplet states of nitrobenzene at a number of characterized critical points. ${ }^{\mathrm{a}}$

\begin{tabular}{|c|c|c|c|c|}
\hline Geometry State & S0 & S1 & $\mathrm{T} 1$ & $\mathrm{~T} 2$ \\
\hline \multicolumn{5}{|c|}{ geometries related to the main decay path ${ }^{\mathrm{b}}$} \\
\hline${ }^{1}(\mathrm{gs})_{\min }{ }^{\mathrm{b}}$ & 0.00 & 3.61 & 3.25 & 3.38 \\
\hline${ }^{1}\left(\mathrm{n}_{\mathrm{A}} \pi^{*}\right)_{\min }^{\mathrm{b}}$ & 0.85 & 2.79 & 2.72 & 3.04 \\
\hline${ }^{3}\left(\mathrm{n}_{\mathrm{A}} \pi^{*}\right)_{\min }^{\mathrm{b}}$ & 0.86 & 2.66 & 2.69 & 3.11 \\
\hline${ }^{3}\left(\pi_{\mathrm{O}} \pi^{*}\right)_{\min }^{\mathrm{b}}$ & 0.52 & 2.93 & 2.94 & 3.04 \\
\hline \multicolumn{5}{|c|}{ geometries characterizing PATH 1, see Figure 2} \\
\hline$(\mathrm{T} 1 / \mathrm{S} 0)_{\mathrm{stc}-\mathrm{NO}}$ & 3.78 & 5.11 & 3.83 & 5.13 \\
\hline \multicolumn{5}{|c|}{ geometries characterizing PATH 2, see Figure 2} \\
\hline$(\mathrm{T} 1 / \mathrm{S} 0)_{\text {mecp }}$ & 3.60 & 3.75 & 3.68 & 3.89 \\
\hline${ }^{1}(\mathrm{~S} 0)_{\mathrm{ep}}$ & 1.81 & 3.55 & 2.70 & 4.74 \\
\hline${ }^{1}(\mathrm{TS})_{\mathrm{ep}}$ & 3.37 & 3.98 & 3.82 & 3.93 \\
\hline \multicolumn{5}{|c|}{ geometries characterizing PATH 3, see Figure 2} \\
\hline$(\mathrm{T} 1 / \mathrm{S} 0)_{\mathrm{roam}}$ & 5.14 & 5.18 & 5.13 & 6.27 \\
\hline${ }^{1}(\mathrm{~S} 0)_{\text {nitrite }}$ & 0.19 & 3.53 & 2.87 & 4.54 \\
\hline${ }^{1}(\mathrm{TS})_{\text {nitrite }}$ & 0.65 & 1.29 & 1.21 & 1.48 \\
\hline
\end{tabular}

${ }^{a}$ All reported energies are referred with respect to the S0 energy at its minimum, ${ }^{1}$ (gs) ${ }_{\min }$.

${ }^{\mathrm{b}}$ Geometry taken from reference 17.
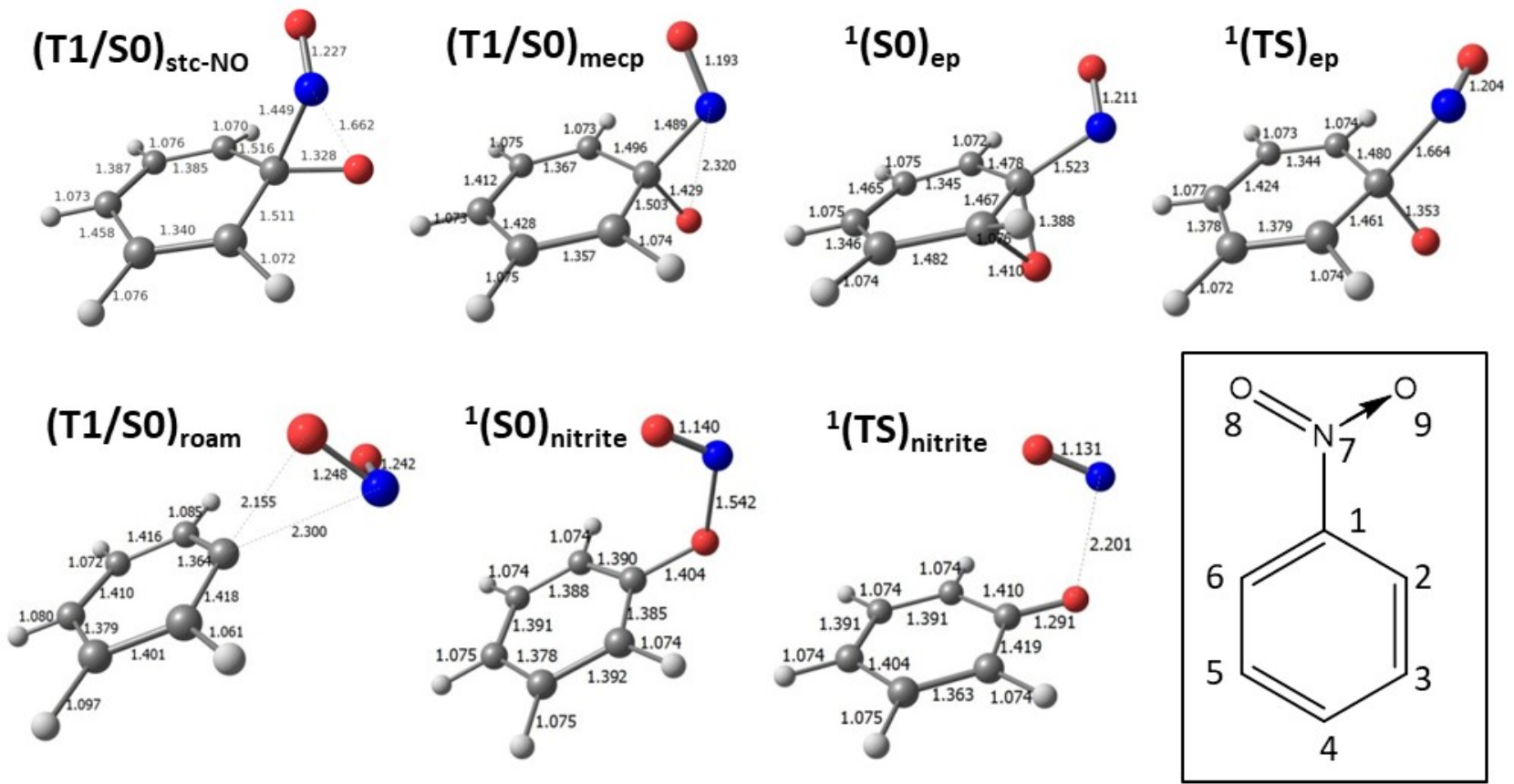

Figure 1. Geometries of nitrobenzene at the characterized critical points. Bonds lengths are reported in $\AA$. In the inset is displayed the nitrobenzene structure and the atom labeling. 

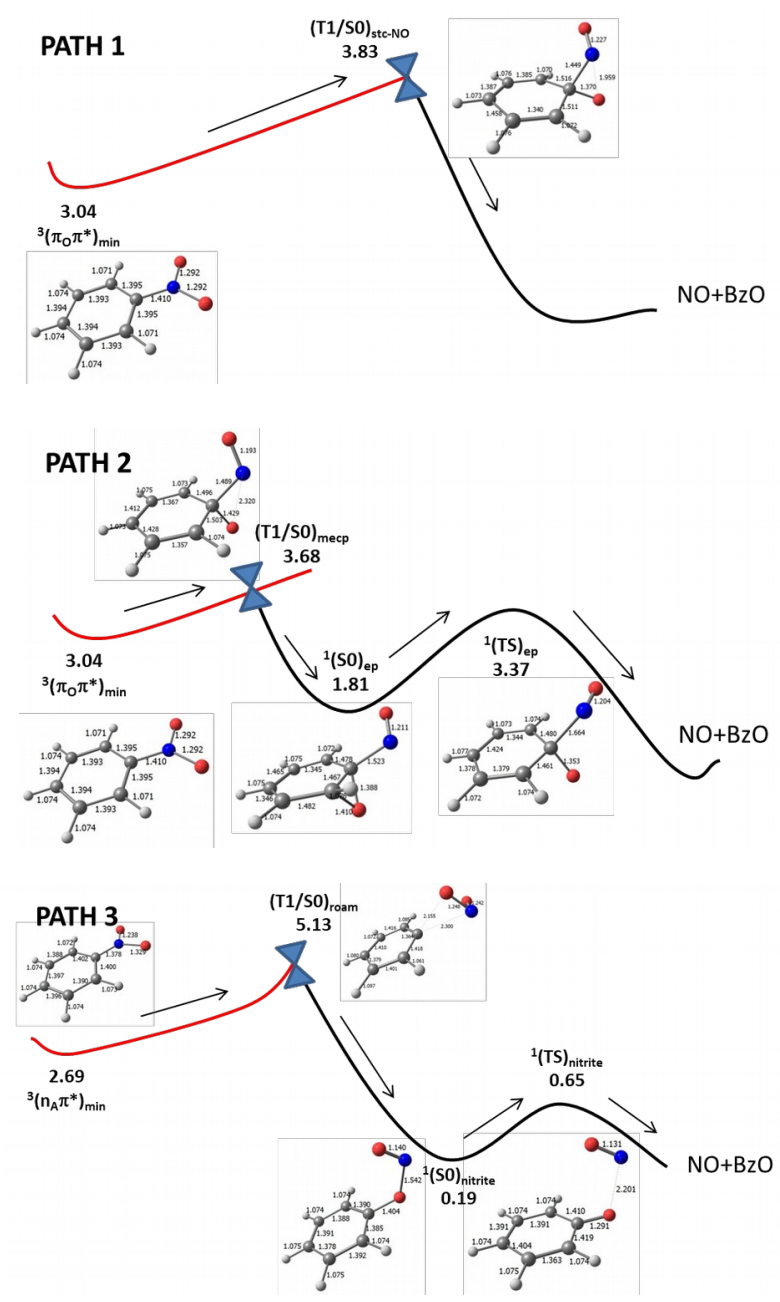

Figure 2. Schematic representation of the three characterized paths leading from excited nitrobenzene to the release of NO. Upper panel: path 1; Middle panel: path 2; Lower panel: path 3.

Suits and co-workers obtained a binomial distribution in the NO translational energy after excitation at $226 \mathrm{~nm}(5.49 \mathrm{eV}),{ }^{14}$ moreover determining a low and high rotational energy for the low and high translational NO products, respectively. Two peaks in the translational energy distribution were characterized, one at around $0.25 \mathrm{eV}$ and the other at around $1 \mathrm{eV}$ (Figure 3 in reference 14). The later peak can be associated with path 1 , while the former peak has a value equally close to both the computed averaged translational energy for path 2 and 3. In this case consequently, the involvement of a roaming mechanism as path 3 is not excluded, which is in agreement with the computed energy of the (T1/S0) roam structure, $5.13 \mathrm{eV}$, placed $0.36 \mathrm{eV}$ below the employed wavelength. A roaming mechanism seems then to be possible, but only at relatively high excitation energies.

In agreement with Suits and co-workers, the here computed averaged rotational energy is higher for path 1, associated with the high translational NO products, than for path 2 and 3, associated with the low translational NO molecules, although the difference is not very remarkable. The higher rotational energy difference is here reported for path 1 and 2 (0.07 eV difference), further proving that the same STC seam can mediate the formation of NO products with different internal energy distributions.

Finally, in agreement with both works and with the data obtained by Galloway at al. ${ }^{11,12}$ performing laser induced fluorescence experiments, in all cases the computed averaged NO vibrational energy is lower than $0.1 \mathrm{eV}$. 
Table 2. Theoretical kinetic $\left(<E_{k i n}>\right)$, translational $\left(<E_{t r}>\right)$, rotational $\left(<E_{\text {rot }}>\right)$, and vibrational energies of the NO molecules computed using CASSCF $(14,11)$ ground state classical dynamics starting from (T1/S0) $)_{\text {stc-No, }}{ }^{1}(\mathrm{TS})_{\mathrm{ep}}$, and ${ }^{1}(\mathrm{TS})_{\text {nitrite }}$ structure, corresponding to the simulation of the NO formation accordingly to path1, path2, and path3, respectively. Experimental energies obtained at different excitation energies are also reported.

\begin{tabular}{|c|c|c|c|c|}
\hline \multicolumn{5}{|c|}{ theoretical energies } \\
\hline $\begin{array}{l}\text { Starting geometry } \\
\text { for the dynamic }\end{array}$ & $<E_{k i n}>$ & $<E_{t r}>$ & $<E_{\text {rot }}>$ & $<E_{v i b}>$ \\
\hline $\begin{array}{l}(\mathrm{T} 1 / \mathrm{S} 0)_{\text {stc-NO }} \\
(\mathrm{PATH} 1)\end{array}$ & $\begin{array}{c}1.39 \mathrm{eV} \\
(32.51 \mathrm{kcal} / \mathrm{mol})\end{array}$ & $\begin{array}{c}1.24 \mathrm{eV} \\
(28.59 \mathrm{kcal} / \mathrm{mol})\end{array}$ & $\begin{array}{c}0.13 \mathrm{eV} \\
(3.00 \mathrm{kcal} / \mathrm{mol})\end{array}$ & $\begin{array}{c}0.02 \mathrm{eV} \\
(0.46 \mathrm{kcal} / \mathrm{mol})\end{array}$ \\
\hline $\begin{array}{l}{ }^{1}(\mathrm{TS})_{\mathrm{ep}} \\
\text { (PATH 2) }\end{array}$ & $\begin{array}{c}0.66 \mathrm{eV} \\
(15.22 \mathrm{kcal} / \mathrm{mol})\end{array}$ & $\begin{array}{c}0.53 \mathrm{eV} \\
(12.22 \mathrm{kcal} / \mathrm{mol})\end{array}$ & $\begin{array}{c}0.06 \mathrm{eV} \\
(1.38 \mathrm{kcal} / \mathrm{mol})\end{array}$ & $\begin{array}{c}0.07 \mathrm{eV} \\
(1.61 \mathrm{kcal} / \mathrm{mol})\end{array}$ \\
\hline $\begin{array}{l}{ }^{1}(\mathrm{TS})_{\text {nitrite }} \\
\text { (PATH 3) }\end{array}$ & $\begin{array}{c}0.13 \mathrm{eV} \\
(\mathrm{kcal} / \mathrm{mol})\end{array}$ & $\begin{array}{c}10^{-6} \mathrm{eV} \\
\left(10^{-5} \mathrm{kcal} / \mathrm{mol}\right)\end{array}$ & $\begin{array}{c}0.10 \mathrm{eV} \\
(2.31 \mathrm{kcal} / \mathrm{mol})\end{array}$ & $\begin{array}{c}0.03 \mathrm{eV} \\
(0.69 \mathrm{kcal} / \mathrm{mol})\end{array}$ \\
\hline \multicolumn{5}{|c|}{ experimental energies } \\
\hline & $E_{\text {kin }}(\mathrm{eV})$ & $E_{t r}(\mathrm{eV})$ & $E_{\text {rot }}(\mathrm{eV})$ & $E_{v i b}(\mathrm{eV})$ \\
\hline $\begin{array}{l}\text { Excitation at } 226 \\
\mathrm{~nm}(5.49 \mathrm{eV})^{11,12}\end{array}$ & - & $1.1 \pm 0.2$ & $0.32 \pm 0.03$ & $<0.1$ \\
\hline $\begin{array}{l}\text { Excitation at } 280 \\
\mathrm{~nm}(4.43 \mathrm{eV})^{11,12}\end{array}$ & - & $0.56 \pm 0.12$ & $0.20 \pm 0.03$ & $<0.1$ \\
\hline $\begin{array}{c}\text { Excitation at } 266 \\
\mathrm{~nm}(4.66 \mathrm{eV})^{44}\end{array}$ & - & $14.4 \%^{\mathrm{a}}$ & $6.1 \%{ }^{\mathrm{a}}$ & $<2.5 \%^{\mathrm{a}}$ \\
\hline $\begin{array}{l}\text { Excitation at } 226 \\
\mathrm{~nm}(5.49 \mathrm{eV})^{14}\end{array}$ & - & $0.025-1.1$ & $0.19-0.76$ & - \\
\hline
\end{tabular}

${ }^{\mathrm{a}}$ Percentage of the total available energy

\section{Conclusions}

Three pathways that lead to the formation of NO from NB have been determined using high-level quantum chemistry calculations. All start on the S0 state after crossing from T1. Two involve isomerization of the nitro group to bond an oxygen to the aromatic ring, with one forming an epoxide. The third path is a roaming mechanism that also leads to isomerization from the nitro to a nitrite group. According to the accepted thinking up to now, roaming is responsible for the formation of low translational and rotational NO photoproducts. As low translational NO molecules are always detected for all excitation energies which result in NO formation, it can be inferred that roaming plays a pivotal role in the NO photoproduction. According to the here present results based on ab-initio CASPT2//CASSCF static and CASSCF classical dynamics calculations, this picture is challenged. Despite confirming the presence of a roaming type mechanism, here characterized in the excited state, which leads to NO with low translational and rotational energy (path 3), the results suggest that this mechanism is operating only at relatively high excitation energies (greater than $5.13 \mathrm{eV}, 242 \mathrm{~nm}$ ). This consequently determines that the roaming mechanism is not responsible for the low translational and rotational NO formation detected between 4.66 and $5.00 \mathrm{eV}$ (266 and 248 $\mathrm{nm}$ ) excitation as reported by Lin et al., so putting into perspective the contribution of roaming in NO production. First, the key geometry leading to roaming, (T1/S0) roam, is placed at $5.13 \mathrm{eV}$ (242 $\mathrm{nm}$ ) with respect to the S0 minimum. Second, a more favorable mechanism also leading to low translational and rotational energy NO photoproducts has been here described (path 2), whose key geometry, (T1/S0) mecp, is placed at $3.60 \mathrm{eV}$ with respect to the $\mathrm{S} 0$ minimum, i.e. $1.53 \mathrm{eV}$ below the (T1/S0) roam. This of course does not exclude the presence of a lower-lying point also promoting the 
roaming mechanism, whose existence, despite the thorough $\mathrm{PEH}$ exploration made here, has yet to be determined.

\section{Acknowledgments}

This project has received funding from the European Union's Horizon 2020 research and innovation programme under the Marie Sklodowska-Curie Grant Agreement No. 658173. A.G. also thanks Spanish MICINN (CTQ2017-87054-C2-2-P), Unidad de Excelencia Maria de Maetzu MDM-20150538, Subprograma Atracció de Talent - Contractes Postdoctorals de la Universitat de València, and Spanish "Juan de la Cierva Incorporación" postdoctoral grant IJC2018-035123-I.

1 T. B. Brill and K. J. James, Kinetics and Mechanisms of Thermal Decomposition of Nitroaromatic explosives, Chem. Rev., 1993, 93, 2667.

2 H. Nakagawa, K. Hishikawa, K. Eto, N. Ieda, T. Namikawa, K. Kamada, T. Suzuki, N. Miyata and J. I. Nabekura, Fine spatiotemporal control of nitric oxide release by infrared pulse-laser irradiation of a photolabile donor, ACS Chem. Biol., 2013, 8, 2493-2500.

3 L. Möller, I. Lax and L. C. Eriksson, Nitrated Polycyclic Aromatic Hydrocarbons: A Risk Assessment for the Urban Citizen, Environ. Heal. Perspect., 1993, 101, 309.

4 R. Morales-Cueto, M. Esquivelzeta-Rabell, J. Saucedo-Zugazagoitia and J. Peon, Singlet Excited-State Dynamics of Nitropolycyclic Aromatic Hydrocarbons: Direct Measurements by Femtosecond Fluorescence Up-Conversion, J. Phys. Chem. A, 2007, 111, 552-557.

5 S. J. Zugazagoitia, C. X. Almora-Díaz and J. Peon, Ultrafast Intersystem Crossing in 1Nitronaphthalene. An Experimental and Computational Study, J. Phys. Chem. A, 2008, 112, 358.

6 J. S. Zugazagoitia, E. Collado-Fregoso, E. F. Plaza-Medina and J. Peon, Relaxation in the triplet manifold of 1-nitronaphthalene observed by transient absorption spectroscopy, $J$. Phys. Chem. A, 2009, 113, 805-810.

7 R. A. Vogt and C. E. Crespo-Hernandez, Conformational Control in the Population of the Triplet State and Photoreactivity of Nitronaphthalene Derivatives, J. Phys. Chem. A, 2013, 117, 14100-14108.

8 O. L. Chapman, D. C. Heckert, J. W. Reasoner and S. P. Thackaberry, Photochemical Studies on 9-Nitroanthracene, J. Am. Chem. Soc., 1966, 88, 5550.

$9 \quad$ K. Fukuhara, M. Kurihara and N. Miyata, Photochemical Generation of Nitric Oxide from 6Nitrobenzo[a]pyrene, J. Am. Chem. Soc., 2001, 123, 8662-8666.

10 E. F. Plaza-medina, W. Rodríguez-c, R. Morales-cueto and J. Peon, Primary Photochemistry of Nitrated Aromatic Compounds : Excited- State Dynamics and NO 3 Dissociation from 9Nitroanthracene, 2011, 115, 577-585.

11 D. B. Galloway, J. A. Bartz, L. G. Huey and F. F. Crim, Pathways and Kinetic Energy Disposal in the Photodissociation of Nitrobenzene, J. Chem. Phys., 1993, 98, 2107.

12 D. B. Galloway, T. Glenewinkel-Meyer, J. a. Bartz, L. G. Huey and F. F. Crim, The kinetic and internal energy of NO from the photodissociation of nitrobenzene, J. Chem. Phys., 1994, 100, 1946. 
13 M.-F. Lin, Y. T. Lee, C.-K. Ni, S. Xu and M. C. Lin, Photodissociation dynamics of nitrobenzene and o-nitrotoluene., J. Chem. Phys., 2007, 126, 064310.

14 M. L. Hause, N. Herath, R. Zhu, M. C. Lin and A. G. Suits, Roaming-mediated isomerization in the photodissociation of nitrobenzene., Nat. Chem., 2011, 3, 932-7.

15 Y. He, A. Gahlmann, J. S. Feenstra, S. T. Park and A. H. Zewail, Ultrafast electron diffraction: structural dynamics of molecular rearrangement in the NO release from nitrobenzene., Chem. Asian J., 2006, 1, 56-63.

16 A. Giussani, Toward the Understanding of the Photophysics and Photochemistry of 1Nitronaphthalene under Solar Radiation: The First Theoretical Evidence of a Photodegradation Intramolecular Rearrangement Mechanism Involving the Triplet States, $J$. Chem. Theory Comput., 2014, 10, 3987-3995.

17 A. Giussani and G. A. Worth, Insights into the Complex Photophysics and Photochemistry of the Simplest Nitroaromatic Compound: A CASPT2//CASSCF Study on Nitrobenzene, J. Chem. Theory Comput., 2017, 13, 2777-2788.

18 J. M. Bowman and B. C. Shepler, Roaming Radicals, Annu. Rev. Phys. Chem., 2011, 62, 531-553.

19 S. A. Lahankar, S. D. Chambreau, D. Townsend, F. Suits, J. Farnum, X. Zhang, J. M. Bowman and A. G. Suits, The roaming atom pathway in formaldehyde decomposition, $J$. Chem. Phys., 2006, 125, 0-10.

20 H. Li, P. Tsai, K. Hung, T. Kasai, K. Lin, H. Li, P. Tsai, K. Hung, T. Kasai and K. Lin, Communication: Photodissociation of $\mathrm{CH} 3 \mathrm{CHO}$ at $308 \mathrm{~nm}$ : Observation of H- roaming, CH3-roaming, and transition state pathways together along the ground state surface, $J$. Chem. Phys., 2015, 142, 041101.

21 M. J. T. Jordan and S. H. Kable, Roaming reaction pathways along excited states, Science (80-. )., 2012, 335, 1054-1055.

22 M. P. Grubb, M. L. Warter, H. Xiao, S. Maeda, K. Morokuma and S. W. North, No straight path: Roaming in both ground- and excited-state photolytic channels of $\mathrm{NO} 3 \rightarrow \mathrm{NO}+02$, Science (80-. )., 2012, 335, 1075-1078.

23 A. Giussani and G. A. Worth, Similar chemical structures, dissimilar triplet quantum yields: A CASPT2 model rationalizing the trend of triplet quantum yields in nitroaromatic systems, Phys. Chem. Chem. Phys., 2019, 21, 10514-10522.

24 S. Nagakura, M. Kojima and Y. Maruyama, Electronic Spectra and Electronic Structures of Nitrobenzene and Nitromesitylene, J. Mol. Spectrosc., 1964, 13, 174.

25 M. Takezaki, N. Hirota and M. Terazima, Nonradiative Relaxation Processes and Electronically Excited States of Nitrobenzene Studied by Picosecond Time-Resolved Transient Grating Method, J. Phys. Chem. A, 1997, 101, 3443.

26 R. Hurley and A. C. Testa, Triplet-State Yield of Aromatic Nitro Compounds, J. Am. Chem. Soc., 1968, 90, 1949. 
K. Andersson, P.-A. Malmqvist and B. O. Roos, Second-order perturbation theory with a complete active space self-consistent field reference function, J. Chem. Phys., 1992, 96, 1218.

28 B. O. Roos, K. Andersson, M. P. Fülscher, P.-Å. Malmqvist, L. Serrano-Andrés, K. Pierloot and M. Merchán, Multiconfigurational Perturbation Theory: Applications in Electronic Spectroscopy, Adv. Chem. Phys., 1996, 93, 219.

29 L. Serrano-Andrés, M. Merchán, P. v. R. Schleyer, P. R. Schreiner, H. F. Schaefer, W. L. Jorgensen, W. Thiel and R. C. Glen, Encyclopedia of Computational Chemistry, 2004.

30 I. Fernández Galván, M. Vacher, A. Alavi, C. Angeli, F. Aquilante, J. Autschbach, J. J. Bao, S. I. Bokarev, N. A. Bogdanov, R. K. Carlson, L. F. Chibotaru, J. Creutzberg, N. Dattani, M. G. Delcey, S. S. Dong, A. Dreuw, L. Freitag, L. M. Frutos, L. Gagliardi, F. Gendron, A. Giussani, L. Gonzalez, G. Grell, M. Guo, C. E. Hoyer, M. Johansson, S. Keller, S. Knecht, G. Kovačević, E. Källman, G. Li Manni, M. Lundberg, Y. Ma, S. Mai, J. P. Malhado, P. A. Malmqvist, P. Marquetand, S. A. Mewes, J. Norell, M. Olivucci, M. Oppel, Q. M. Phung, K. Pierloot, F. Plasser, M. Reiher, A. M. Sand, I. Schapiro, P. Sharma, C. J. Stein, L. K. Sørensen, D. G. Truhlar, M. Ugandi, L. Ungur, A. Valentini, S. Vancoillie, V. Veryazov, O. Weser, T. A. Wesolowski, P.-O. Widmark, S. Wouters, A. Zech, J. P. Zobel and R. Lindh, OpenMolcas: From source code to insight, J. Chem. Theory Comput., 2019, 15, 5925-5964.

31 P.-O. Widmark, P.-Å. Malmqvist and B. O. Roos, Density Matrix Averaged Atomic Natural Orbital (ANO) Basis Sets for Correlated Molecular Wave Functions, Theor. Chem. Acc., 1990, 77, 291.

32 K. Pierloot, B. Dumez, P.-O. Widmark and B. O. Roos, Density Matrix Averaged Atomic Natural Orbital (ANO) Basis Sets for Correlated Molecular Wave Functions, Theor. Chim. Acta, 1995, 90, 87.

33 J.-M. Mewes, V. Jovanović, C. M. Marian and A. Dreuw, On the Molecular Mechanism of Non-Radiative Decay of Nitrobenzene and the Unforeseen Challenges this Simple Molecule Holds for Electronic Structure Theory, Phys. Chem. Chem. Phys., 2014, 16, 12393.

34 M. Merchán, L. Serrano-Andrés and M. Olivucci, Computational Photochemistry, Elsevier, Amsterdam, 1st edn., 2005, vol. 16.

35 A. Giussani, R. Pou-Amérigo, L. Serrano-Andrés, A. Freire-Corbacho, C. Martínez-García, M. I. Fernández P, M. Sarakha, M. Canle L and J. A. Santaballa, Combined theoretical and experimental study of the photophysics of asulam., J. Phys. Chem. A, 2013, 117, 2125-37.

36 A. Giussani, L. Serrano-Andrés, M. Merchán, D. Roca-Sanjuán and M. Garavelli, Photoinduced Formation Mechanism of the Thymine-Thymine (6-4) Adduct, J. Phys. Chem. B, 2013, 117, 1999.

37 I. F. Galván, M. G. Delcey, T. B. Pedersen, F. Aquilante and R. Lindh, Analytical StateAverage Complete-Active-Space Self-Consistent Field Nonadiabatic Coupling Vectors: Implementation with Density-Fitted Two-Electron Integrals and Application to Conical Intersections, J. Chem. Theory Comput., 2016, 12, 3636-3653. 
38 J. M. Del Campo and A. M. Köster, A hierarchical transition state search algorithm, J. Chem. Phys., 2008, 129, 024107.

39 M. Merchán, L. Serrano-Andrés, M. A. Robb and L. Blancafort, Triplet-State Formation along the Ultrafast Decay of Excited Singlet Cytosine, J. Am. Chem. Soc., 2005, 127, 1820.

40 T. B. Pedersen, R. Lindh and F. Aquilante, Density fitting with auxiliary basis sets from Cholesky decompositions, Theor. Chem. Acc., 2009, 124, 1.

41 The (T1/S0) $)_{\text {stc-No }}$ was obtained in reference 17 extracting along CASSCF MECP optimization one of the structure displaying S0-T1 degeneracy at the CASPT2(16,13) level.

42 H. J. Wörner, J. B. Bertrand, B. Fabre, J. Higuet, H. Ruf, A. Dubrouil, S. Patchkovskii, M. Spanner, Y. Mairesse, V. Blanchet, E. Mével, E. Constant, P. B. Corkum and D. M. Villeneuve, Conical Intersection Dynamics in NO2 Probed by Homodyne High-Harmonic Spectroscopy, Science (80-. )., 2011, 334, 208-212.

43 G. Olaso-González, D. Roca-Sanjuán, L. Serrano-Andŕs and M. Merchán, Toward the understanding of DNA fluorescence: The singlet excimer of cytosine, J. Chem. Phys., 2006, 125, 1-5.

44 Y.-M. Li, J.-L. Sun, H.-M. Yin, K.-L. Han and G.-Z. He, Photodissociation of nitrobenzene at $266 \mathrm{~nm}$ : Experimental and theoretical approach, J. Chem. Phys., 2003, 118, 6244. 